\title{
Prior ankle fractures in postmenopausal women are associated with low areal bone mineral density and bone microstructure alterations
}

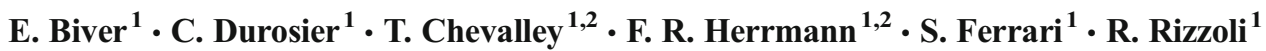

Received: 19 December 2014 / Accepted: 23 March 2015 /Published online: 8 April 2015

(C) International Osteoporosis Foundation and National Osteoporosis Foundation 2015

\begin{abstract}
Summary In a cross-sectional analysis in postmenopausal women, prior ankle fractures were associated with lower areal bone mineral density (BMD) and trabecular bone alterations compared to no fracture history. Compared to women with forearm fractures, microstructure alterations were of lower magnitude. These data suggest that ankle fractures are another manifestation of bone fragility.

Introduction Whether ankle fractures represent fragility fractures associated with low areal bone mineral density (aBMD) and volumetric bone mineral density (vBMD) and/or bone microstructure alterations remains unclear, in contrast to the well-recognised association between forearm fractures and osteoporosis. The objective of this study was to investigate aBMD, vBMD and bone microstructure in postmenopausal women with prior ankle fracture in adulthood, compared with women without prior fracture or with women with prior forearm fractures, considered as typically of osteoporotic origin. Methods In a cross-sectional analysis in the Geneva Retirees Cohort study, 63 women with ankle fracture and 59 with forearm fracture were compared to 433 women without fracture (mean age, $65 \pm 1$ years). aBMD was measured by dual-energy $\mathrm{X}$-ray absorptiometry; distal radius and tibia vBMD and bone
\end{abstract}

E. Biver

Emmanuel.Biver@hcuge.ch

1 Division of Bone Diseases, Department of Internal Medicine Specialties, Faculty of Medicine, Geneva University Hospitals, 4 Rue Gabrielle Perret-Gentil, 1211 Geneva 14, Switzerland

2 Division of Geriatrics, Department of Internal Medicine, Rehabilitation and Geriatrics, Faculty of Medicine, Geneva University Hospitals, 4 Rue Gabrielle Perret-Gentil, 1211 Geneva 14, Switzerland microstructure were measured by high-resolution peripheral quantitative computed tomography.

Results Compared with women without fracture, those with ankle fractures had lower aBMD, radius vBMD $(-7.9 \%)$, trabecular density $(-10.7 \%)$, number $(-7.3 \%)$ and thickness $(-4.6 \%)$ and higher trabecular spacing $(+14.5 \%)(P<0.05$ for all). Tibia trabecular variables were also altered. For 1 standard deviation decrease in total hip aBMD or radius trabecular density, odds ratios for ankle fractures were 2.2 and 1.6 , respectively, vs 2.2 and 2.7 for forearm fracture, respectively $(P \leq 0.001$ for all). Compared to women with forearm fractures, those with ankle fractures had similar spine and hip aBMD, but microstructure alterations of lower magnitude. Conclusion Women with ankle fractures have lower aBMD and $v B M D$ and trabecular bone alterations, suggesting that ankle fractures are another manifestation of bone fragility.

Keywords Ankle fracture $\cdot \mathrm{HR}-\mathrm{pQCT} \cdot$ Microstructure Osteoporosis $\cdot$ Volumetric BMD

\section{Introduction}

Ankle and forearm fractures are common non-vertebral fractures and their incidence increases with age [1]. Whilst forearm fractures are classically regarded as of bone fragility (osteoporotic) origin, it remains debated whether ankle fractures can be considered as osteoporotic fragility fractures. Ankle fractures are associated with particular risk factors, such as higher body mass index, a history of falls and a higher degree of physical activity $[2,3]$. A study among US Medicare beneficiaries indicated that ankle fractures are associated with prior forearm, humerus and femoral fractures and increase the risk of subsequent fragility spine and forearm fractures [4]. Previous osteoporotic fractures were shown also to 
increase the risk of ankle fracture in perimenopausal women [5]. Conversely, data are conflicting regarding their potential association with low areal bone mineral density (aBMD) $[2$, 6-8]. One study in 17 women with a history of ankle fracture after menopause did not show a marked decrease in aBMD, but some microstructure alterations [9]. In the Study of Osteoporotic Fractures in which fracture risk was prospectively assessed in women aged 65 years and older over an average of 8.5 years after spine and hip aBMD measurements, the risk of ankle fracture was not significantly associated with low aBMD, in contrast to forearm fractures $[10,11]$. Other longitudinal studies have indicated that a prior ankle fracture did not predict [12-14] or only weakly predicted [1] subsequent osteoporotic fractures in women. However, the risk was no longer significant 1 to 5 years after the fracture [1].

In the context of the debated issue to consider ankle fractures like another osteoporotic fracture, we investigated in postmenopausal women aged $65 \pm 1.4$ years the association between ankle fractures and bone microstructure alterations using highresolution peripheral quantitative computed tomography (HRpQCT). Microstructure alteration is an aBMD-independent determinant of fracture risk that contributes to bone mechanical properties $[15,16]$, and HR-pQCT measurement results allow the discrimination between osteopenic women with and without prior fragility fractures [17]. The results were compared with those observed in subjects with forearm fractures, taken as positive controls. As forearm fractures are generally recognised as osteoporotic fractures, identifying similar alterations of bone mineral density (BMD) and bone microstructure in women with prior ankle fracture and women with prior forearm fracture would provide a rationale to consider ankle fractures as osteoporotic fractures as well.

\section{Subjects and methods}

\section{Study design and participants}

We conducted a cross-sectional study in the Geneva Retirees Cohort (GERICO), a cohort of 953 healthy men and women who were recruited between 2008 and 2010 in Geneva (Switzerland) at the time of retirement, i.e. at 65 years, by advertisement in local newspapers or among the staff of Geneva University Hospitals and local large companies. All subjects signed a written informed consent before undergoing a medical interview and a series of measurements to investigate the genetic, environmental and nutritional determinants of fractures. Dietary calcium and protein intake, as well as physical activity, was assessed using frequency questionnaires as previously described $[18,19]$. Body weight and standing height were measured, and body mass index (BMI) was calculated. Tobacco (current vs never or past) and alcohol ( $\geq 7$ units weekly vs less) consumption was assessed as well as age at menarche and menopause and current or past drugs use, with a specific focus on bone-targeted treatments. Participants in the present study are the 749 postmenopausal women included at baseline in this cohort. The study protocol was approved by the ethics committee of the Geneva University Hospitals.

\section{Fracture assessment}

Fracture history since birth was retrospectively recorded during an interview, in which all subjects were asked if they had ever suffered a fracture, on details on fracture site; age at time of fracture; type and intensity of trauma associated with the fracture; and modalities of fracture treatment. Fractures of the skull, toes and fingers were excluded. Only fractures that occurred in adulthood (after the age of 20 years) were taken into account in the analyses, and fractures were divided for subgroup analyses according to age at time of fracture (before or after the age of 45). Regarding ankle fractures, proximal or medial tibia/fibula fractures and calcaneus/tarsus fractures were differentiated during the interview from malleolar fractures to exclude potential non-malleolar fractures reported as ankle fractures by the subjects. However, due to the lack of systematic X-rays, we were unable to better characterise fracture types among the heterogeneity of the actual ankle fracture classifications (medial/lateral malleolus fractures, bimalleolar/trimalleolar fractures).

\section{Areal bone mineral density measurements}

aBMD $\left(\mathrm{g} / \mathrm{cm}^{2}\right)$ of the lumbar spine (L1-4), non-dominant femoral neck, total hip, distal third, ultra-distal and total radius was measured using dual-energy X-ray absorptiometry (DXA) scanning equipment (Hologic QDR Discovery instrument; Hologic Inc., Waltham, MA), located in a mobile unit. The coefficient of variation (CV) of repeated measurements varied between 1.0 and $1.5 \%$. Osteoporotic status was defined as osteoporosis if the spine or hip T-score was $\leq-2.5$ standard deviation (SD), osteopenia if the spine or hip T-score was between -1 and $-2.5 \mathrm{SD}$ and normal if the spine or hip Tscore was $>-1 \mathrm{SD}$, using National Health and Nutrition Examination Survey (NHANES) III references for proximal femur BMD and the Hologic database for spine BMD.

\section{HR-pQCT measurements}

Volumetric bone mineral density (vBMD) and microstructure variables were determined within the same week of DXA at the distal radius and tibia by HR-pQCT using an Xtreme CT instrument (Scanco Medical, Brüttisellen, Switzerland). A stack of $110 \mathrm{CT}$ slices were acquired over a $9 \mathrm{~mm}$ length with an isotropic voxel size of $82 \mu \mathrm{m}$, starting proximally at 9.5 and $22.5 \mathrm{~mm}$ from a joint margin reference line for distal radius and distal tibia, respectively. The effective dose was $3 \mu \mathrm{Sv}$, and the measurement time was $2.8 \mathrm{~min}$. Short-term reproducibility 
assessed with repositioning was 0.6-1.0 and 2.8-4.9\% for density variables and trabecular microstructure, respectively. Determinations were performed on the non-dominant limb, unless a fracture was reported in the region of interest. Recorded variables were as follows: total, cortical and trabecular vBMD, expressed in milligrams per cubic centimetre $\left(\mathrm{mg} / \mathrm{cm}^{3}\right)$ of calcium hydroxyapatite; total cross-sectional area and cortical and trabecular areas $\left(\mathrm{mm}^{2}\right)$; relative trabecular bone volume (BV/TV) $(\%)$; trabecular number $\left(\mathrm{mm}^{-1}\right)$, thickness and spacing $(\mu \mathrm{m})$; trabecular spacing $\mathrm{SD}$, as an estimate of the heterogeneity of the trabecular structure $(\mu \mathrm{m})$; and mean cortical thickness $(\mu \mathrm{m})$. Cortical porosity $(\%)$ was calculated as the number of void voxels in each binary cortex image divided by the total number of voxels [20].

\section{Statistical analysis}

The various anthropometric, BMD and microstructure variables were compared between women with ankle fractures and women without fracture as well as between women with ankle fractures and women with forearm fracture, taken as a fragility fracture referent group. To check if the well-known association between forearm fractures and low $\mathrm{aBMD}$ and bone microstructure alterations was reproduced in our cohort, variables were also compared between women with forearm fractures and women without fracture. Data are presented as means $\pm \mathrm{SD}$ or number and percentage. Normality testing (Shapiro-Francia $W$ test and Skewness-Kurtosis tests) was performed, and non-Gaussian variables were normalised using simple mathematical transformations. Differences in density, microstructure and clinical characteristics between groups were assessed using an analysis of covariance with normalised variables to control for the influence of age, height, weight, years since menopause (including menopausal hormone therapy duration), calcium and protein intake and physical activity. A chi-square test with a threshold of $5 \%$ was used to test the difference for qualitative variables between fractured and nonfractured subjects. Associations between density, microstructure parameters and fracture status were evaluated by logistic regression analysis with adjustment for age, height, weight, years since menopause, calcium and protein intake and physical activity and were expressed as odds ratio (OR) with $95 \%$ confidence intervals (CIs) for a BMD or a microstructure parameter change of 1 SD. Two-sided $P<0.05$ was considered to indicate statistical significance for all tests. Analyses were conducted with Stata version 12.1 (StataCorp LP, College Station, TX).

\section{Results}

\section{Characteristics of the subjects according to fracture history}

The characteristics of the 749 postmenopausal women included in the GERICO cohort are shown in Table 1. Five hundred and thirty-one fractures were reported by 316 (42\%) women, including 63 ankle fractures and 59 forearm fractures in adulthood ( 8.3 and $7.9 \%$, respectively). Age at time of fracture (median, interquartile range) was 54 (44-59) years for ankle fractures and 57 (47-62) years for forearm fractures. Seven women had experienced both ankle and forearm fractures in adulthood. Four hundred and thirty-three women did not report any fracture. Anthropometric parameters, calcium and protein dietary intake, physical activity and tobacco and alcohol use did not differ between women with ankle or forearm fracture compared with those without fracture history (Table 1). There was no difference in the prevalence of obesity and type 2 diabetes between groups. Women with ankle fracture had their menopause approximately 2 years earlier than women without fracture $(P=0.007)$, and the age at menarche was 7 and 8 months higher in women with ankle and forearm fractures ( $P=0.002$ and 0.001 , respectively). The use of calcium and vitamin D supplements, menopausal hormone therapy and anti-osteoporotic drugs did not differ between women with ankle or forearm fracture and those without fracture history. Only one woman in the ankle fracture group was treated with alendronate; none in the forearm fracture group received any anti-osteoporotic drug. Three women (5\%) had previously received an anti-osteoporotic drug in each fracture group [ankle fracture group: alendronate (two) and risedronate (one); forearm fracture group: alendronate (two) and raloxifene (one)] as well as $22(5.1 \%)$ in the non-fractured group.

\section{Ankle fractures are associated with lower aBMD and bone microstructure alterations}

The risks for having sustained any other fractures in adulthood (after the age of 20 years), as well as fragility fractures after the age of 45 years, were increased to the same extent in the presence of a prior ankle or forearm fracture having occurred in adulthood [OR (95\% CI) 2.08 (1.24-3.50), $P=0.006$, and 1.98 (1.16-3.37), $P=0.012$, respectively, for any other fractures in adulthood; 1.89 (1.04-3.44), $P=0.037$, and 2.16 (1.18-3.97), $P=0.012$, respectively, for after 45 years of having fragility fractures]. Based on this similar ratio, we determined vBMD and bone microstructure alterations in women with prior ankle fracture.

aBMD was lower at all sites in women with ankle fractures than in those without fracture. Differences observed were $7 \%$ for lumbar spine BMD, $5.4 \%$ for femoral neck BMD, $5.1 \%$ for total hip BMD, $4.6 \%$ for radius total BMD, $3.9 \%$ for distal third radius and $5.4 \%$ for ultra-distal radius BMD $(P<0.01$ for all) (Table 2, Fig. 1). These differences remained statistically significant after adjustment for several potential confounding variables including age, years since menopause, standing height, body weight, calcium and protein intake and physical activity (Table 2). Based on NHANES III references for proximal femur BMD and the Hologic database for spine 


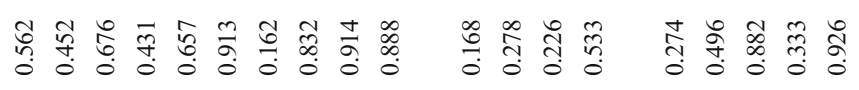

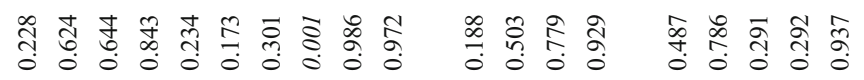

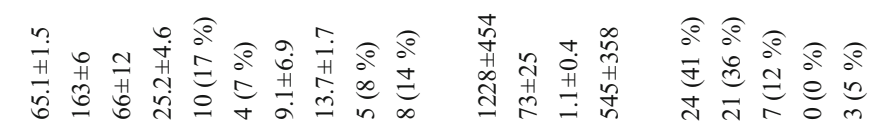

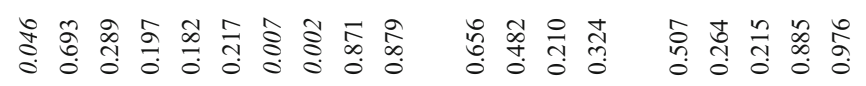

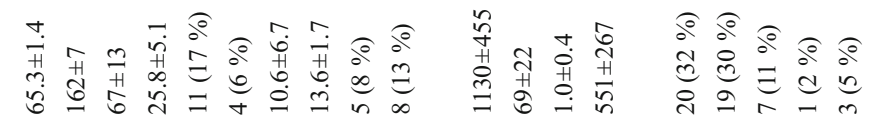

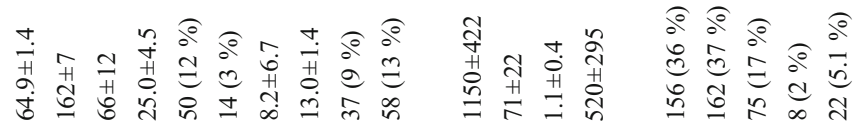

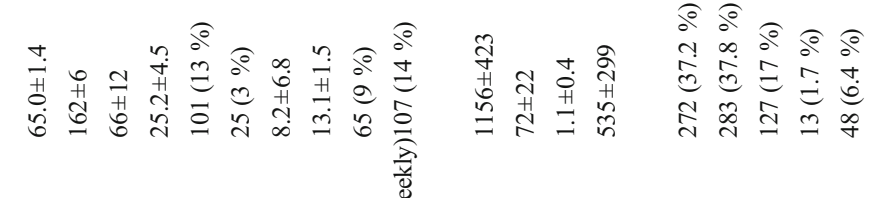


BMD, 25 and $16 \%$ of women were osteoporotic in the ankle fracture group and control group, respectively $(P=0.007)$.

Compared to women without fractures, women with a prior ankle fracture had lower distal radius total vBMD (-7.9\%), trabecular bone density $(-10.7 \%)$, trabecular number (Tb.N) $(-7.3 \%)$ and trabecular thickness (Tb.Th) $(-4.6 \%)(P<0.05$ for all). Adjustment for the above-mentioned potential confounding factors did not modify these results (Table 2, Fig. 1). trabecular spacing (Tb.Sp) (+14.5\%) and distribution $(+32 \%)$ were higher among women with ankle fractures $(P<0.01)$. After controlling for radial one-third distal aBMD, radius trabecular vBMD and distribution remained significantly different between the two groups ( $P=0.050$ and 0.041 , respectively), indicating that some changes in microstructure were not captured in aBMD. Distal tibia trabecular microstructure was also altered in the ankle fracture group (Tb.N, $-5.6 \%$; Tb.Sp, $+8.9 \%$; and distribution, $+19.5 \%, P<0.05$ for all) (Table 2, Fig. 1).

\section{Compared to forearm fractures, ankle fractures are associated with similar spine and hip aBMD and bone microstructure alterations of lower magnitude}

As previously reported, women with a forearm fracture had lower aBMD and bone microstructure alterations (Table 2, Fig. 1). When ankle and forearm fractures were compared, there was no difference in osteoporotic status $(P=0.333)$ nor in aBMD, except for ultra-distal radius BMD that tended to be lower in the forearm fracture group $(-10.1 \mathrm{vs}-5.4 \%, P=$ 0.056 and 0.025 after multiple adjustments). Some alterations of bone microstructure were of lower magnitude in women with ankle fracture vs forearm fracture, including distal radius trabecular area $(+1.1 \mathrm{vs}+12 \%, P=0.007)$, tibia total vBMD $(-3.4$ vs $-13.1 \%, P=0.005)$, trabecular area $(-1.7 \mathrm{vs}+8.4 \%$, $P=0.004)$, vBMD ( -5 vs $-13.9 \%, P=0.037)$, Tb. Th $(+1.4$ vs $-8.1 \%, P=0.003)$ and cortical thickness $(-3.1$ vs $-13.1 \%$, $P=0.027$ ) (Fig. 1).

\section{Ankle and forearm fracture risk according to aBMD and microstructure parameters}

We then evaluated the independent contribution of bone mass/ density and microstructure in ankle and forearm fracture risk (OR adjusted for age, years since menopause, height, weight, calcium and protein intake and physical activity). Prior ankle or forearm fractures were not influenced by body weight or BMI assessed at the age of 65 years ( $\mathrm{OR}=1$ for both, $P=0.289$ and 0.644 for every $5-\mathrm{kg}$ increase in body weight $/ 5 \mathrm{~kg} / \mathrm{m}^{2}$ in $\mathrm{BMI}$, respectively). One SD reduction in lumbar spine or hip aBMD was associated with a higher risk of prior ankle or forearm fractures (Table 3). Ankle fractures were associated with lower distal radius total vBMD and all trabecular parameters, but not cortical vBMD, distal tibia trabecular density, number, spacing and distribution. Forearm fractures were associated with lower distal radius aBMD and lower distal radius and tibia microstructure parameters. Distal radius trabecular vBMD was the bone parameter whose variations were the most associated with ankle or forearm fracture prevalence [OR (95 \% CI) 1.6 (1.2-2.2), $P=0.001$, and 2.7 (1.9-3.9), $P<0.001$, respectively]. ORs were lower for ankle fracture than forearm fracture for ultra-distal radius aBMD $(P=$ $0.024)$, distal radius total and trabecular vBMD $(P=0.027$ and 0.049$)$, distal tibia total and cortical vBMD $(P=0.008$ and 0.039$)$ and cortical and Tb.Th $(P=0.027$ and 0.019$)$ (Table 3).

The influence of age at the time of fractures was also investigated. Ankle fractures were associated with distal radius trabecular vBMD both when the fracture occurred between 20 and 44 years of age [OR $(95 \% \mathrm{CI}) 2.03$ (1.14-3.63), $P=$ 0.016 ] or after the age of 45 years [OR (95\% CI) 1.44 (1.01-2.04), $P=0.044]$. For forearm fracture, the association was mainly observed when the fracture occurred after the age of 45 years [OR $(95 \%$ CI) 3.3 (2.08-5.22), $P=<0.001$, vs 1.92 (1-3.7), $P=0.051$, for fracture between 20 and 44 years].

\section{Discussion}

Whilst forearm fractures are well-recognised fragility osteoporotic fractures, thus associated with lower aBMD, it remains debated whether ankle fractures fulfil the same criteria [10, 11]. This plays an important role in the perspective of secondary fracture prevention. Indeed, the occurrence of forearm fracture is an indication to secondary prevention, whilst ankle fracture would also become an indication in the case of being recognised as a fragility osteoporotic fracture $[10,11]$. In this study, our results show that women at the age of $65 \pm 1.4$ years who experienced an ankle fracture in adulthood have a twofold higher risk of having sustained any other prior fractures or fragility fractures than those without any ankle fracture history. This ratio is similar to that observed in women who experienced a forearm fracture in adulthood. The association of ankle fractures with other fractures was previously reported in a study including 29,802 respondents to a postal inquiry in Norway [21]. In addition, the prevalence of major osteoporotic fractures was associated with prior ankle fractures in the Manitoba cohort [adjusted OR (95 \% CI) 1.40 (1.13-1.75), $P=0.002$ ] [14].

To investigate the determinants of the associations of ankle and/or forearm fractures with other fractures, we compared aBMD and bone microstructure according to fracture history. Women at the age of 65 years who had experienced an ankle fracture in adulthood had lower spine and proximal femur aBMD, lower distal radius vBMD and bone microstructure alterations at distal radius and tibia that predominate on the trabecular bone compartment compared to women without a 
Table 2 Areal and volumetric BMD and bone microstructure in postmenopausal women according to their fracture history

\begin{tabular}{|c|c|c|c|c|c|c|c|c|c|}
\hline & \multirow{2}{*}{$\begin{array}{l}\text { No fracture } \\
N=433\end{array}$} & \multirow{2}{*}{$\begin{array}{l}\text { Ankle fracture } \\
N=63\end{array}$} & \multicolumn{2}{|c|}{ Ankle vs no fracture } & \multirow{2}{*}{$\begin{array}{l}\text { Forearm fracture } \\
N=59\end{array}$} & \multicolumn{2}{|c|}{ Forearm vs no fracture } & \multicolumn{2}{|c|}{ Ankle vs forearm fractur } \\
\hline & & & $P^{\mathrm{a}}$ & $P^{\mathrm{ab}}$ & & $P^{\mathrm{c}}$ & $P^{\mathrm{bc}}$ & $P^{\mathrm{d}}$ & $P^{\mathrm{bd}}$ \\
\hline \multicolumn{10}{|l|}{ Areal BMD $\left(\mathrm{g} / \mathrm{cm}^{2}\right)$} \\
\hline Lumbar spine & $0.935 \pm 0.152$ & $0.870 \pm 0.155$ & $<0.001$ & $<0.001$ & $0.854 \pm 0.136$ & $<0.001$ & $<0.001$ & 0.616 & 0.592 \\
\hline Femoral neck & $0.717 \pm 0.106$ & $0.678 \pm 0.114$ & 0.003 & $<0.001$ & $0.670 \pm 0.104$ & $<0.001$ & $<0.001$ & 0.754 & 0.998 \\
\hline Total hip & $0.855 \pm 0.112$ & $0.811 \pm 0.107$ & 0.002 & $<0.001$ & $0.803 \pm 0.114$ & $<0.001$ & $<0.001$ & 0.656 & 0.888 \\
\hline Distal $1 / 3$ radius & $0.639 \pm 0.065$ & $0.614 \pm 0.067$ & 0.004 & 0.009 & $0.619 \pm 0.057$ & 0.025 & 0.013 & 0.604 & 0.820 \\
\hline Ultra-distal radius & $0.404 \pm 0.060$ & $0.382 \pm 0.063$ & 0.007 & 0.007 & $0.363 \pm 0.049$ & $<0.001$ & $<0.001$ & 0.056 & 0.025 \\
\hline Total radius & $0.534 \pm 0.059$ & $0.509 \pm 0.062$ & 0.002 & 0.003 & $0.502 \pm 0.046$ & $<0.001$ & $<0.001$ & 0.493 & 0.307 \\
\hline \multicolumn{10}{|l|}{ Osteoporotic status ${ }^{\mathrm{e}}, n(\%)$} \\
\hline $\begin{array}{l}\text { Osteoporosis } \\
\text { Osteopenia }\end{array}$ & $\begin{array}{l}70(16) \\
246(57)\end{array}$ & $\begin{array}{l}16(25) \\
39(62)\end{array}$ & 0.007 & 0.002 & $\begin{array}{l}22(37) \\
29(49)\end{array}$ & $<0.001$ & $<0.001$ & 0.333 & 0.368 \\
\hline Normal BMD & $116(27)$ & $8(13)$ & & & $8(14)$ & & & & \\
\hline \multicolumn{10}{|l|}{ Radius HR-pQCT } \\
\hline Total vBMD $\left(\mathrm{mg} \mathrm{HA} / \mathrm{cm}^{3}\right)$ & $303 \pm 66$ & $279 \pm 62$ & 0.008 & 0.013 & $257 \pm 60$ & $<0.001$ & $<0.001$ & 0.064 & 0.030 \\
\hline Cortical vBMD (mg HA/cm $\left.{ }^{3}\right)$ & $866 \pm 64$ & $853 \pm 64$ & 0.156 & 0.396 & $839 \pm 76$ & 0.012 & 0.032 & 0.338 & 0.136 \\
\hline Trabecular vBMD (mg HA/ $\left./ \mathrm{cm}^{3}\right)$ & $143 \pm 37$ & $127 \pm 43$ & 0.004 & 0.004 & $113 \pm 32$ & $<0.001$ & $<0.001$ & 0.051 & 0.053 \\
\hline $\mathrm{BV} / \mathrm{TV}(\%)$ & $0.119 \pm 0.031$ & $0.106 \pm 0.036$ & 0.004 & 0.004 & $0.094 \pm 0.026$ & $<0.001$ & $<0.001$ & 0.051 & 0.053 \\
\hline Tb.N $\left(\mathrm{mm}^{-1}\right)$ & $1.818 \pm 0.321$ & $1.686 \pm 0.387$ & 0.007 & 0.005 & $1.597 \pm 0.363$ & $<0.001$ & $<0.001$ & 0.160 & 0.129 \\
\hline $\operatorname{Tb} . T h(\mu \mathrm{m})$ & $0.065 \pm 0.011$ & $0.062 \pm 0.013$ & 0.024 & 0.033 & $0.059 \pm 0.009$ & $<0.001$ & $<0.001$ & 0.201 & 0.237 \\
\hline $\mathrm{Tb} . \mathrm{Sp}(\mu \mathrm{m})$ & $0.509 \pm 0.161$ & $0.583 \pm 0.272$ & 0.008 & 0.006 & $0.608 \pm 0.192$ & $<0.001$ & $<0.001$ & 0.107 & 0.084 \\
\hline Tb.SpSD $(\mu \mathrm{m})$ & $0.250 \pm 0.189$ & $0.330 \pm 0.343$ & 0.005 & 0.003 & $0.354 \pm 0.261$ & $<0.001$ & $<0.001$ & 0.180 & 0.073 \\
\hline Ct.Th (mm) & $0.729 \pm 0.177$ & $0.681 \pm 0.166$ & 0.051 & 0.103 & $0.647 \pm 0.180$ & 0.003 & 0.004 & 0.315 & 0.156 \\
\hline $\mathrm{CSA}\left(\mathrm{mm}^{2}\right)$ & $257 \pm 44$ & $257 \pm 35$ & 0.823 & 0.953 & $280 \pm 45$ & 0.001 & 0.003 & 0.006 & 0.003 \\
\hline Cortical area $\left(\mathrm{mm}^{2}\right)$ & $49 \pm 11$ & $46 \pm 11$ & 0.057 & 0.114 & $45 \pm 12$ & 0.032 & 0.026 & 0.787 & 0.432 \\
\hline Trabecular area $\left(\mathrm{mm}^{2}\right)$ & $203 \pm 44$ & $205 \pm 34$ & 0.593 & 0.728 & $227 \pm 47$ & $<0.001$ & 0.001 & 0.007 & 0.003 \\
\hline Cortical porosity (\%) & $2.7 \pm 1.4$ & $2.7 \pm 1.9$ & 0.526 & 0.280 & $2.5 \pm 1.4$ & 0.278 & 0.152 & 0.724 & 0.805 \\
\hline Ct.Pm $(\mu \mathrm{m})$ & $68 \pm 6$ & $68 \pm 5$ & 0.611 & 0.656 & $71 \pm 6$ & $<0.001$ & 0.002 & 0.008 & 0.006 \\
\hline \multicolumn{10}{|l|}{ Tibia HR-pQCT } \\
\hline Total vBMD (mg HA/ $\left./ \mathrm{cm}^{3}\right)$ & $260 \pm 51$ & $251 \pm 52$ & 0.195 & 0.181 & $226 \pm 42$ & $<0.001$ & $<0.001$ & 0.005 & 0.008 \\
\hline Cortical vBMD (mg HA $\left./ \mathrm{cm}^{3}\right)$ & $822 \pm 60$ & $816 \pm 61$ & 0.471 & 0.803 & $799 \pm 56$ & 0.006 & 0.007 & 0.098 & 0.043 \\
\hline Trabecular vBMD (mg HA/ $\left.\mathrm{cm}^{3}\right)$ & $152 \pm 36$ & $145 \pm 37$ & 0.113 & 0.057 & $131 \pm 25$ & $<0.001$ & $<0.001$ & 0.037 & 0.083 \\
\hline BV/TV (\%) & $0.127 \pm 0.030$ & $0.121 \pm 0.030$ & 0.113 & 0.057 & $0.109 \pm 0.021$ & $<0.001$ & $<0.001$ & 0.037 & 0.083 \\
\hline Tb.N $\left(\mathrm{mm}^{-1}\right)$ & $1.717 \pm 0.316$ & $1.620 \pm 0.341$ & 0.031 & 0.009 & $1.634 \pm 0.287$ & 0.061 & 0.028 & 0.796 & 0.907 \\
\hline $\operatorname{Tb} . \operatorname{Th}(\mu \mathrm{m})$ & $0.074 \pm 0.014$ & $0.075 \pm 0.013$ & 0.786 & 0.831 & $0.068 \pm 0.013$ & $<0.001$ & $<0.001$ & 0.003 & 0.020 \\
\hline $\mathrm{Tb} . \mathrm{Sp}(\mu \mathrm{m})$ & $0.530 \pm 0.122$ & $0.577 \pm 0.181$ & 0.035 & 0.011 & $0.565 \pm 0.117$ & 0.015 & 0.006 & 0.820 & 0.761 \\
\hline Tb.SpSD $(\mu \mathrm{m})$ & $0.261 \pm 0.142$ & $0.312 \pm 0.236$ & 0.021 & 0.009 & $0.282 \pm 0.126$ & 0.034 & 0.015 & 0.845 & 0.977 \\
\hline Ct.Th $(\mu \mathrm{m})$ & $0.959 \pm 0.234$ & $0.929 \pm 0.250$ & 0.377 & 0.593 & $0.833 \pm 0.239$ & $<0.001$ & $<0.001$ & 0.027 & 0.026 \\
\hline $\mathrm{CSA}\left(\mathrm{mm}^{2}\right)$ & $693 \pm 109$ & $679 \pm 102$ & 0.373 & 0.266 & $735 \pm 106$ & 0.007 & 0.004 & 0.004 & 0.003 \\
\hline Cortical area $\left(\mathrm{mm}^{2}\right)$ & $98 \pm 21$ & $94 \pm 22$ & 0.171 & 0.327 & $89 \pm 20$ & 0.002 & 0.001 & 0.164 & 0.130 \\
\hline Trabecular area $\left(\mathrm{mm}^{2}\right)$ & $586 \pm 114$ & $576 \pm 109$ & 0.542 & 0.408 & $635 \pm 114$ & 0.003 & 0.001 & 0.004 & 0.003 \\
\hline Cortical porosity (\%) & $8.3 \pm 3.1$ & $7.9 \pm 2.9$ & 0.370 & 0.178 & $8.8 \pm 3.5$ & 0.258 & 0.355 & 0.132 & 0.061 \\
\hline Ct.Pm $(\mu \mathrm{m})$ & $103 \pm 9$ & $102 \pm 8$ & 0.453 & 0.369 & $113 \pm 54$ & 0.002 & $<0.001$ & 0.006 & 0.009 \\
\hline
\end{tabular}

Values are means \pm SD or number $(\%)$

Tb.SpSD trabecular spacing standard deviation, Ct.Th cortical thickness, Ct.Pm cortical perimeter

$P$-values in italics: statistically significant

${ }^{a} P$ value of the differences between women with ankle fracture and without fracture

${ }^{\mathrm{b}} P$ value after adjustment for age, height, weight, years since menopause, calcium and protein intake and physical activity

${ }^{\mathrm{c}} P$ value of the differences between women with forearm fracture and without fracture

${ }^{\mathrm{d}} P$ value of the differences between women with ankle fracture and forearm fracture

${ }^{\mathrm{e}}$ At the spine, femoral neck or total hip 
Fig. 1 Differences in mean aBMD and microstructure variables at the distal radius and tibia between women with ankle or forearm fracture and those without fracture. ${ }^{*} P<0.05$; + $P<0.01 ; \ddagger P<0.001$. The $P$ value of the difference between women with ankle and forearm fractures is indicated at the foot of each column pair. NS not significant
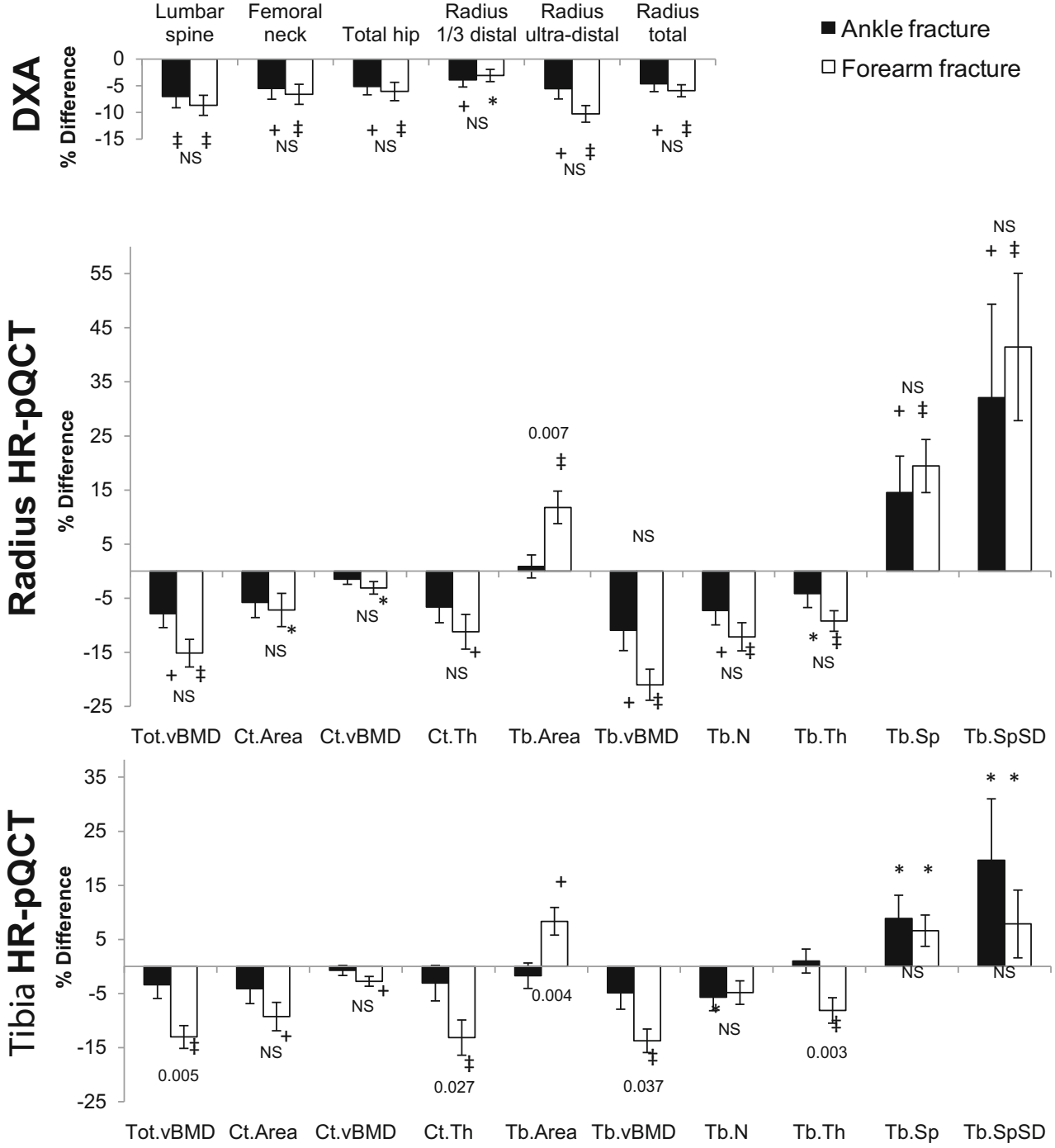

fracture history. In agreement with a previous study focused on ankle fractures occurring after the menopause [9], we found trabecular bone alterations, which were not captured by DXA. However, we did not detect any alteration in the cortical bone compartment. The higher menopause duration and later age at menarche may contribute to trabecular bone alteration observed in women with ankle fractures, as we previously demonstrated [22]. In addition, higher lifelong physical activity in women with ankle fractures might provide a protective effect on tibia cortical bone. Indeed, a positive relationship between high-impact activity and cortical bone has been shown, particularly in subjects with high fat mass, another classically reported risk factor for ankle fractures [23]. As cortical bone represents a high proportion of bone mass, this observation may contribute to apparent relatively preserved aBMD observed in previous studies, despite alterations of the trabecular bone compartment.

As mentioned, body weight is a risk factor of ankle fracture, which is partially captured in aBMD [24]. Indeed, when compared with non-obese patients, obese patients are more likely to fracture their ankle, but less likely to fracture their wrist [25]. In the Global Longitudinal Study of Osteoporosis in Women (GLOW), body weight showed a significant positive association with ankle fractures, but a negative association with forearm fractures [24]. Although we did not detect any difference in body weight and prevalence of obesity between ankle-fractured and fracture-free subjects, all data were adjusted for weight and height. The risk of prior ankle or forearm fractures was not influenced by body weight or BMI assessed at the time of BMD or microstructure measurements. Lifestyle habits, diet and physical activity may, however, have changed body weight since fracture occurrence.

We then compared aBMD, vBMD and bone microstructure in women with a prior ankle fracture vs those with a prior forearm fracture. As previously reported [26], women with a prior forearm fracture had lower aBMD and altered bone microstructure compared with those without a prior fracture. However, alterations in women with prior ankle fracture were 
Table 3 Risk of ankle or forearm fracture per $1 \mathrm{SD}$ decrease (increase for Tb.Sp and Tb.SpSD) in aBMD or microstructure variables at the distal radius and tibia

\begin{tabular}{|c|c|c|c|c|c|}
\hline & \multicolumn{2}{|l|}{ Ankle fracture } & \multicolumn{2}{|l|}{ Forearm fracture } & \multirow{2}{*}{$\begin{array}{l}\text { Ankle vs forearm } \\
\text { fracture } \\
P\end{array}$} \\
\hline & OR $(95 \% \mathrm{CI})$ & $P$ & OR $(95 \% \mathrm{CI})$ & $P$ & \\
\hline \multicolumn{6}{|l|}{ Areal BMD (DXA) } \\
\hline Lumbar spine & $1.92(1.42-2.60)$ & $<0.001$ & $2.02(1.48-2.76)$ & $<0.001$ & 0.560 \\
\hline Femoral neck & $1.97(1.42-2.74)$ & $<0.001$ & $2.05(1.45-2.88)$ & $<0.001$ & 0.999 \\
\hline Total hip & $2.16(1.54-3.03)$ & $<0.001$ & $2.19(1.55-3.08)$ & $<0.001$ & 0.876 \\
\hline Distal $1 / 3$ radius & $1.60(1.20-2.13)$ & 0.001 & $1.45(1.09-1.93)$ & 0.01 & 0.806 \\
\hline Ultra-distal radius & $1.62(1.21-2.17)$ & 0.001 & $2.37(1.71-3.29)$ & $<0.001$ & 0.024 \\
\hline Total radius & $1.69(1.26-2.27)$ & $<0.001$ & $1.93(1.42-2.63)$ & $<0.001$ & 0.289 \\
\hline \multicolumn{6}{|l|}{ Radius (HR-pQCT) } \\
\hline Total vBMD & 1.55 (1.15-2.09) & 0.004 & $2.24(1.59-3.15)$ & $<0.001$ & 0.027 \\
\hline $\mathrm{Ct}$ vBMD & $1.24(0.93-1.65)$ & 0.150 & $1.43(1.05-1.96)$ & 0.024 & 0.122 \\
\hline Ct.Th & $1.38(1.02-1.85)$ & 0.034 & $1.62(1.17-2.26)$ & 0.004 & 0.139 \\
\hline Tb vBMD & $1.64(1.21-2.21)$ & 0.001 & $2.70(1.86-3.92)$ & $<0.001$ & 0.049 \\
\hline Tb.N & $1.60(1.19-2.17)$ & 0.002 & $2.15(1.53-3.02)$ & $<0.001$ & 0.118 \\
\hline Tb.Th & $1.41(1.06-1.88)$ & 0.019 & $1.95(1.40-2.73)$ & $<0.001$ & 0.221 \\
\hline Tb.Sp & $1.44(1.15-1.81)$ & 0.001 & $1.55(1.20-2.01)$ & 0.001 & 0.079 \\
\hline Tb.SpSD & $1.38(1.11-1.71)$ & 0.004 & $1.42(1.11-1.82)$ & 0.006 & 0.069 \\
\hline \multicolumn{6}{|l|}{ Tibia (HR-pQCT) } \\
\hline Total vBMD & $1.32(0.98-1.80)$ & 0.071 & $2.28(1.64-3.18)$ & $<0.001$ & 0.008 \\
\hline $\mathrm{Ct}$ vBMD & $1.18(0.87-1.60)$ & 0.287 & $1.57(1.15-2.14)$ & 0.005 & 0.039 \\
\hline Ct.Th & $1.21(0.89-1.66)$ & 0.229 & $1.91(1.37-2.67)$ & $<0.001$ & 0.027 \\
\hline $\mathrm{Tb}$ vBMD & $1.35(1.01-1.80)$ & 0.045 & $1.96(1.44-2.66)$ & $<0.001$ & 0.074 \\
\hline Tb.N & $1.53(1.12-2.09)$ & 0.007 & $1.41(1.05-1.91)$ & 0.024 & 0.915 \\
\hline Tb.Th & $0.98(0.74-1.30)$ & 0.882 & $1.66(1.24-2.23)$ & 0.001 & 0.019 \\
\hline Tb.Sp & $1.48(1.14-1.92)$ & 0.003 & $1.36(1.05-1.76)$ & 0.02 & 0.737 \\
\hline Tb.SpSD & $1.33(1.07-1.65)$ & 0.009 & $1.15(0.92-1.45)$ & 0.223 & 0.992 \\
\hline
\end{tabular}

OR $(95 \% \mathrm{CI})$ was adjusted for age, height, weight, years since menopause, calcium and protein intake and physical activity (logistic regression after normalisation of non-normally distributed variables)

$\nu B M D$ volumetric bone mineral density, $C t$ cortical, $C t$.Th cortical thickness, $T b$ trabecular, $T b . N$ trabecular number, $T b . T h$ trabecular thickness, $T b . S p$ trabecular spacing, $T b . S p S D$ trabecular spacing standard deviation

$P$-values in italics: statistically significant of lower magnitude than in those with prior forearm fractures, especially for distal radius trabecular area, distal tibia total vBMD, trabecular vBMD, area and Tb.Th and cortical thickness. These data may suggest that other determinants contribute to ankle fracture risk in addition to aBMD and bone microstructure alterations. In contrast to forearm fractures, this could mitigate the association between low aBMD at baseline and the risk of ankle fracture in longitudinal studies [10, 11].

The strengths of our study are first an age-homogenous group of healthy postmenopausal women recruited at a mean age of $65 \pm 1.4$ years when fracture risk markedly increases in the general population. Second, the analysis of ankle and forearm fractures was conducted in relation to a large variety of different bone mass, density and microstructure variables. Third, low prevalence of anti-osteoporotic drugs in subjects with previous forearm or ankle fractures reflects the inclusion criteria of this study that recruited healthy participants, but it highlights also the treatment gap of osteoporosis in postmenopausal women with prior fragility fractures [27]. Finally, the analysis of ankle-fractured subjects in comparison to those with forearm fractures allowed us to validate the accuracy of the data of our cohort by reproducing the well-known association between forearm fractures and low aBMD and bone microstructure alterations, as previously reported [26]. In addition, it provided the opportunity to compare the level of associations of ankle and forearm fractures with bone parameter alterations. The main limitations of this study are the cross-sectional design, the retrospective documentation of fractures based on interview and the lack of consistently collected X-rays to validate fracture type. The possible influence of fracture on bone microstructure was limited by performing the HR-pQCT scans on the non-dominant limb and on the 
contra-lateral one if a wrist or ankle fracture was reported in the region of interest.

In conclusion, postmenopausal women at the age of $65 \pm$ 1.4 years who had experienced an ankle fracture or a forearm fracture in adulthood had similar twofold higher prevalence of other fractures. Women with prior ankle or forearm fractures had similar alterations of spine and hip aBMD. However, the alterations in the trabecular compartment were of lower magnitude in the ankle compared to the forearm fracture group. The alterations of aBMD, vBMD and bone microstructure observed in women with prior ankle fractures provide a rationale for considering ankle fractures, like forearm fractures, as fragility osteoporotic fractures. These data argue for the inclusion of ankle fracture history in fracture risk assessment and as an indication for secondary fracture prevention.

Acknowledgments We thank Ms. F. Merminod, RD; M.-A. Schaad, $\mathrm{RN}$; and A. Sigaud, RN, for the management of participants; Ms. C. Genet and Mr. G. Conicella for DXA and HR-pQCT measurements; and Ms. R. Sudan for manuscript editing. We thank the Swiss Foundation for Research in Ageing AETAS for the kind supply of its mobile osteodensitometer, the Geneva University Hospitals and Faculty of Medicine Clinical Research Center and the BNP-Paribas Foundation for their support.

Conflicts of interest None

\section{References}

1. Giangregorio LM, Leslie WD (2010) Time since prior fracture is a risk modifier for 10-year osteoporotic fractures. J Bone Miner Res 25:1400-1405

2. Seeley DG, Kelsey J, Jergas M, Nevitt MC (1996) Predictors of ankle and foot fractures in older women. The Study of Osteoporotic Fractures Research Group. J Bone Miner Res 11:1347-1355

3. Hasselman CT, Vogt MT, Stone KL, Cauley JA, Conti SF (2003) Foot and ankle fractures in elderly white women. Incidence and risk factors. J Bone Joint Surg Am 85-A:820-824

4. Taylor AJ, Gary LC, Arora T et al (2011) Clinical and demographic factors associated with fractures among older Americans. Osteoporos Int 22:1263-1274

5. Valtola A, Honkanen R, Kroger H, Tuppurainen M, Saarikoski S, Alhava E (2002) Lifestyle and other factors predict ankle fractures in perimenopausal women: a population-based prospective cohort study. Bone 30:238-242

6. Greenfield DM, Eastell R (2001) Risk factors for ankle fracture. Osteoporos Int 12:97-103

7. Ingle BM, Eastell R (2002) Site-specific bone measurements in patients with ankle fracture. Osteoporos Int 13:342-347

8. Lee KM, Chung CY, Kwon SS, Won SH, Lee SY, Chung MK, Park MS (2013) Ankle fractures have features of an osteoporotic fracture. Osteoporos Int 24:2819-2825

9. Stein EM, Liu XS, Nickolas TL et al (2011) Abnormal microarchitecture and stiffness in postmenopausal women with ankle fractures. J Clin Endocrinol Metab 96:2041-2048

10. Delmas PD, Marin F, Marcus R, Misurski DA, Mitlak BH (2007) Beyond hip: importance of other nonspinal fractures. Am J Med 120:381-387
11. Stone KL, Seeley DG, Lui LY, Cauley JA, Ensrud K, Browner WS, Nevitt MC, Cummings SR (2003) BMD at multiple sites and risk of fracture of multiple types: long-term results from the Study of Osteoporotic Fractures. J Bone Miner Res 18:1947-1954

12. Lauritzen JB, Lund B (1993) Risk of hip fracture after osteoporosis fractures. 451 women with fracture of lumbar spine, olecranon, knee or ankle. Acta Orthop Scand 64:297-300

13. Center JR, Bliuc D, Nguyen TV, Eisman JA (2007) Risk of subsequent fracture after low-trauma fracture in men and women. JAMA 297:387-394

14. Pritchard JM, Giangregorio LM, Ioannidis G, Papaioannou A, Adachi JD, Leslie WD (2012) Ankle fractures do not predict osteoporotic fractures in women with or without diabetes. Osteoporos Int 23:957-962

15. Boutroy S, Bouxsein ML, Munoz F, Delmas PD (2005) In vivo assessment of trabecular bone microarchitecture by highresolution peripheral quantitative computed tomography. J Clin Endocrinol Metab 90:6508-6515

16. Stein EM, Liu XS, Nickolas TL et al (2010) Abnormal microarchitecture and reduced stiffness at the radius and tibia in postmenopausal women with fractures. J Bone Miner Res 25: 2572-2581

17. Nicks KM, Amin S, Atkinson EJ, Riggs BL, Melton LJ 3rd, Khosla S (2012) Relationship of age to bone microstructure independent of areal bone mineral density. J Bone Miner Res 27:637-644

18. Morin P, Herrmann F, Ammann P, Uebelhart B, Rizzoli R (2005) A rapid self-administered food frequency questionnaire for the evaluation of dietary protein intake. Clin Nutr 24:768-774

19. Chevalley T, Bonjour JP, van Rietbergen B, Ferrari S, Rizzoli R (2013) Fracture history of healthy premenopausal women is associated with a reduction of cortical microstructural components at the distal radius. Bone 55:377-383

20. Nishiyama KK, Macdonald HM, Buie HR, Hanley DA, Boyd SK (2010) Postmenopausal women with osteopenia have higher cortical porosity and thinner cortices at the distal radius and tibia than women with normal aBMD: an in vivo HR-pQCT study. J Bone Miner Res 25:882-890

21. Gunnes M, Mellstrom D, Johnell O (1998) How well can a previous fracture indicate a new fracture? A questionnaire study of 29,802 postmenopausal women. Acta Orthop Scand 69:508-512

22. Chevalley T, Bonjour JP, van Rietbergen B, Rizzoli R, Ferrari S (2012) Fractures in healthy females followed from childhood to early adulthood are associated with later menarcheal age and with impaired bone microstructure at peak bone mass. J Clin Endocrinol Metab 97:4174-81

23. Deere K, Sayers A, Rittweger J, Tobias JH (2012) A cross-sectional study of the relationship between cortical bone and high-impact activity in young adult males and females. J Clin Endocrinol Metab 97:3734-3743

24. Compston JE, Flahive J, Hosmer DW et al (2014) Relationship of weight, height, and body mass index with fracture risk at different sites in postmenopausal women: the Global Longitudinal study of Osteoporosis in Women (GLOW). J Bone Miner Res 29:487-493

25. Ong T, Sahota O, Tan W, Marshall L (2014) A United Kingdom perspective on the relationship between body mass index (BMI) and bone health: a cross sectional analysis of data from the Nottingham Fracture Liaison Service. Bone 59:207-210

26. Melton LJ 3rd, Riggs BL, van Lenthe GH, Achenbach SJ, Muller R, Bouxsein ML, Amin S, Atkinson EJ, Khosla S (2007) Contribution of in vivo structural measurements and load/strength ratios to the determination of forearm fracture risk in postmenopausal women. J Bone Miner Res 22:1442-1448

27. Svedbom A, Ivergard M, Hernlund E, Rizzoli R, Kanis JA (2014) Epidemiology and economic burden of osteoporosis in Switzerland. Arch Osteoporos 9:187. doi:10.1007/s11657-0140187-y 